\title{
Computing Optimal Descriptions for Optimality Theory Grammars with Context-Free Position Structures
}

\author{
Bruce Tesar \\ The Rutgers Center for Cognitive Science / \\ The Linguistics Department \\ Rutgers University \\ Piscataway, NJ 08855 USA \\ tesar@ruccs.rutgers.edu
}

\begin{abstract}
This paper describes an algorithm for computing optimal structural descriptions for Optimality Theory grammars with context-free position structures. This algorithm extends Tesar's dynamic programming approach (Tesar, 1994) (Tesar, 1995a) to computing optimal structural descriptions from regular to context-free structures. The generalization to contextfree structures creates several complications, all of which are overcome without compromising the core dynamic programming approach. The resulting algorithm has a time complexity cubic in the length of the input, and is applicable to grammars with universal constraints that exhibit context-free locality.
\end{abstract}

\section{Computing Optimal Descriptions in Optimality Theory}

In Optimality Theory (Prince and Smolensky, 1993), grammaticality is defined in terms of optimization. For any given linguistic input, the grammatical structural description of that input is the description, selected from a set of candidate descriptions for that input, that best satisfies a ranked set of universal constraints. The universal constraints often conflict: satisfying one constraint may only be possible at the expense of violating another one. These conflicts are resolved by ranking the universal constraints in a strict dominance hierarchy: one violation of a given constraint is strictly worse than any number of violations of a lower-ranked constraint. When comparing two descriptions, the one which better satisfies the ranked constraints has higher Harmony. Cross-linguistic variation is accounted for by differences in the ranking of the same constraints.

The term linguistic input should here be understood as something like an underlying form. In phonology, an input might be a string of segmental material; in syntax, it might be a verb's argument structure, along with the arguments. For expositional purposes, this paper will assume linguistic inputs to be ordered strings of segments. A candidate structural description for an input is a full linguistic description containing that input, and indicating what the (pronounced) surface realization is. An important property of Optimality Theory (OT) grammars is that they do not accept or reject inputs; every possible input is assigned a description by the grammar.

The formal definition of Optimality Theory posits a function, Gen, which maps an input to a large (often infinite) set of candidate structural descriptions, all of which are evaluated in parallel by the universal constraints. An OT grammar does not itself specify an algorithm, it simply assigns a grammatical structural description to each input. However, one can ask the computational question of whether efficient algorithms exist to compute the description assigned to a linguistic input by a grammar.

The most apparent computational challenge is posed by the allowance of faithfulness violations: the surface form of a structural description may not be identical with the input. Structural positions not filled with input segments constitute overparsing (epenthesis). Input segments not parsed into structural positions do not appear in the surface pronunciation, and constitute underparsing (deletion). To the extent that underparsing and overparsing are avoided, the description is said to be faithful to the input. Crucial to Optimality Theory are faithfulness constraints, which are violated by underparsing and overparsing. The faithfulness constraints ensure that a grammar will only tolerate deviations of the surface form from the input form which are necessary to satisfy structural constraints dominating the faithfulness constraints.

Computing an optimal description means considering a space of candidate descriptions that include structures with a variety of faithfulness violations, and evaluating those candidates with respect to a ranking in which structural and faithfulness constraints may be interleaved. This is parsing in the generic sense: a structural description is being as- 
signed to an input. It is, however, distinct from what is traditionally thought of as parsing in computational linguistics. Traditional parsing attempts to construct a grammatical description with a surface form matching the given input string exactly; if a description cannot be fit exactly, the input string is rejected as ungrammatical. Traditional parsing can be thought of as enforcing faithfulness absolutely, with no faithfulness violations are allowed. Partly for this reason, traditional parsing is usually understood as mapping a surface form to a description. In the computation of optimal descriptions considered here, a candidate that is fully faithful to the input may be tossed aside by the grammar in favor of a less faithful description better satisfying other (dominant) constraints. Computing an optimal description in Optimality Theory is more naturally thought of as mapping an underlying form to a description, perhaps as part of the process of language production.

Tesar (Tesar, 1994) (Tesar, 1995a) has developed algorithms for computing optimal descriptions, based upon dynamic programming. The details laid out in (Tesar, 1995a) focused on the case where the set of structures underlying the Gen function are formally regular. In this paper, Tesar's basic approach is adopted, and extended to grammars with a Gen function employing fully context-free structures. Using such context-free structures introduces some complications not apparent with the regular case. This paper demonstrates that the complications can be dealt with, and that the dynamic programming case may be fully extended to grammars with context-free structures.

\section{Context-Free Position Structure Grammars}

Tesar (Tesar, 1995a) formalizes Gen as a set of matchings between an ordered string of input segments and the terminals of each of a set of position structures. The set of possible position structures is defined by a formal grammar, the position structure grammar. A position structure has as terminals structural positions. In a valid structural description, each structural position may be filled with at most one input segment, and each input segment may be parsed into at most one position. The linear order of the input must be preserved in all candidate structural descriptions.

This paper considers Optimality Theory grammars where the position structure grammar is context-free; that is, the space of position structures can be described by a formal context-free grammar. As an illustration, consider the grammar in Examples 1 and 2 (this illustration is not intended to represent any plausible natural language theory, but does use the "peak/margin" terminology sometimes employed in syllable theories). The set of inputs is $\{\mathrm{C}, \mathrm{V}\}^{+}$. The candidate descriptions of an input consist of a sequence of pieces, each of which has a peak (p) surrounded by one or more pairs of margin positions $(\mathrm{m})$. These structures exhibit prototypical context-free behavior, in that margin positions to the left of a peak are balanced with margin positions to the right. ' $e$ ' is the empty string, and ' $\mathrm{S}$ ' the start symbol.

\section{Example 1 The Position Structure Grammar}

$$
\begin{aligned}
& \mathrm{S} \Rightarrow \mathrm{F} \mid \mathrm{e} \\
& \mathrm{F} \Rightarrow \mathrm{Y} \mid \mathrm{YF} \\
& \mathrm{Y} \Rightarrow \mathrm{P} \mid \mathrm{MFM} \\
& \mathrm{M} \Rightarrow \mathrm{m} \\
& \mathrm{P} \Rightarrow \mathrm{p}
\end{aligned}
$$

\begin{tabular}{|c|c|}
\hline$-(\mathrm{m} / \mathrm{V})$ & arse $\mathrm{V}$ int \\
\hline$-(\mathrm{p} / \mathrm{C})$ & position \\
\hline PARsE & Input segments must be parsed \\
\hline FILL $^{m}$ & must be filled \\
\hline FILL $^{p}$ & iust be filled \\
\hline
\end{tabular}

\section{Example 2 The Constraints}

The first two constraints are structural, and mandate that $\mathrm{V}$ not be parsed into a margin position, and that $\mathrm{C}$ not be parsed into a peak position. The other three constraints are faithfulness constraints. The two structural constraints are satisfied by descriptions with each $\mathrm{V}$ in a peak position surrounded by matched C's in margin positions: CCVCC, $\mathrm{V}$, CVCCCVCC, etc. If the input string permits such an analysis, it will be given this completely faithful description, with no resulting constraint violations (ensuring that it will be optimal with respect to any ranking).

Consider the constraint hierarchy in Example 3.

\section{Example 3 A Constraint Hierarchy}

$$
\{-(\mathrm{m} / \mathrm{V}),-(\mathrm{p} / \mathrm{C}), \mathrm{PARSE}\} \gg\left\{\mathrm{F}_{\mathrm{ILL}}{ }^{p}\right\} \gg\left\{\mathrm{FILL}^{m}\right\}
$$

This ranking ensures that in optimal descriptions, a $\mathrm{V}$ will only be parsed as a peak, while a $\mathrm{C}$ will only be parsed as a margin. Further, all input segments will be parsed, and unfilled positions will be included only as necessary to produce a sequence of balanced structures. For example, the input /VC/ receives the description ${ }^{1}$ shown in Example 4.

\section{Example 4 The Optimal Description for /VC/$$
\mathrm{S}(\mathrm{F}(\mathrm{Y}(\mathrm{M}(\mathcal{C}), \mathrm{P}(\mathrm{V}), \mathrm{M}(\mathrm{C}))))
$$

The surface string for this description is $\mathcal{C V C}$ : the first $\mathcal{C}$ was "epenthesized" to balance with the one following the peak $\mathrm{V}$. This candidate is optimal because it only violates FILL ${ }^{m}$, the lowest-ranked constraint.

Tesar identifies locality as a sufficient condition on the universal constraints for the success of his

\footnotetext{
${ }^{1}$ In this paper, tree structures will be denoted with parentheses: a parent node $X$ with child nodes $Y$ and $Z$ is denoted $\mathrm{X}(\mathrm{Y}, \mathrm{Z})$.
} 
approach. For formally regular position structure grammars, he defines a local constraint as one which can be evaluated strictly on the basis of two consecutive positions (and any input segments filling those positions) in the linear position structure. That idea can be extended to the context-free case as follows. A local constraint is one which can be evaluated strictly on the basis of the information contained within a local region. A local region of a description is either of the following:

- a non-terminal and the child non-terminals that it immediately dominates;

- a non-terminal which dominates a terminal symbol (position), along with the terminal and the input segment (if present) filling the terminal position.

It is important to keep clear the role of the position structure grammar. It does not define the set of grammatical structures, it defines the space of candidate structures. Thus, the computation of descriptions addressed in this paper should be distinguished from robust, or error-correcting, parsing (Anderson and Backhouse, 1981, for example). There, the input string is mapped to the grammatical structure that is 'closest'; if the input completely matches a structure generated by the grammar, that structure is automatically selected. In the OT case presented here, the full grammar is the entire OT system, of which the position structure grammar is only a part. Error-correcting parsing uses optimization only with respect to the faithfulness of pre-defined grammatical structures to the input. OT uses optimization to define grammaticality.

\section{The Dynamic Programming Table}

The Dynamic Programming (DP) Table is here a three-dimensional, pyramid-shaped data structure. It resembles the tables used for context-free chart parsing (Kay, 1980) and maximum likelihood computation for stochastic context-free grammars (Lari and Young, 1990) (Charniak, 1993). Each cell of the table contains a partial description (a part of a structural description), and the Harmony of that partial description. A partial description is much like an edge in chart parsing, covering a contiguous substring of the input. A cell is identified by three indices, and denoted with square brackets (e.g., $[\mathrm{X}, \mathrm{a}, \mathrm{c}]$ ). The first index identifying the cell $(\mathrm{X})$ indicates the cell category of the cell. The other two indices ( $a$ and $c$ ) indicate the contiguous substring of the input string covered by the partial description contained in the cell (input segments $i_{a}$ through $i_{c}$ ).

In chart parsing, the set of cell categories is precisely the set of non-terminals in the grammar, and thus a cell contains a subtree with a root nonterminal corresponding to the cell category, and with leaves that constitute precisely the input substring covered by the cell. In the algorithm presented here, the set of cell categories are the non-terminals of the position structure grammar, along with a category for each left-aligned substring of the right hand side of each position grammar rule. Example 5 gives the set of cell categories for the position structure grammar in Example 1.

\section{Example 5 The Set of Cell Categories}

$$
\text { S, F, Y, M, P, MF }
$$

The last category in Example 5, MF, comes from the rule $\mathrm{Y} \Rightarrow \mathrm{MFM}$ of Example 1, which has more than two non-terminals on the right hand side. Each such category corresponds to an incomplete edge in normal chart parsing; having a table cell for each such category eliminates the need for a separate data structure containing edges. The cell $[\mathrm{MF}, \mathrm{a}, \mathrm{c}]$ may contain an ordered pair of subtrees, the first with root $M$ covering input $[a, b]$, and the second with root $F$ covering input $[b+1, c]$.

The DP Table is perhaps best envisioned as a set of layers, one for each category. A layer is a set of all cells in the table indexed by a particular cell category.

Example 6 A Layer of the Dynamic Programming Table for Category $M$ (input $i_{1}-i_{3}$ )

\begin{tabular}{|c|c|c|}
\hline$[M, 1,3]$ & & \\
\hline$[\mathrm{M}, 1,2]$ & {$[\mathrm{M}, 2, \overline{3}]$} & \\
\hline $\mathrm{M}, 1,1$ & {$[\mathrm{M}, 2,2]$} & {$[\overline{\mathrm{M}}, \mathbf{3}, 3]$} \\
\hline
\end{tabular}

For each substring length, there is a collection of rows, one for each category, which will collectively be referred to as a level. The first level contains the cells which only cover one input segment; the number of cells in this level will be the number of input segments multiplied by the number of cell categories. Level two contains cells which cover input substrings of length two, and so on. The top level contains one cell for each category. One other useful partition of the DP table is into blocks. A block is a set of all cells covering a particular input subsequence. A block has one cell for each cell category.

A cell of the DP Table is filled by comparing the results of several operations, each of which try to fill a cell. The operation producing the partial description with the highest Harmony actually fills the cell. The operations themselves are discussed in Section 4.

The algorithm presented in Section 6 fills the table cells level by level: first, all the cells covering only one input segment are filled, then the cells covering two consecutive segments are filled, and so forth. When the table has been completely filled, cell $[S, 1, \mathrm{~J}]$ will contain the optimal description of the input, and its Harmony. The table may also be filled in a more left-to-right manner, bottom-up, in the spirit of CKY. First, the cells covering only segment $i_{1}$, and then $i_{2}$, are filled. Then, the cells 
covering the first two segments are filled, using the entries in the cells covering each of $i_{1}$ and $i_{2}$. The cells of the next diagonal are then filled.

\section{The Operations Set}

The Operations Set contains the operations used to fill DP Table cells. The algorithm proceeds by considering all of the operations that could be used to fill a cell, and selecting the one generating the partial description with the highest Harmony to actually fill the cell. There are three main types of operations, corresponding to underparsing, parsing, and overparsing actions. These actions are analogous to the three primitive actions of sequence comparison (Sankoff and Kruskal, 1983): deletion, correspondence, and insertion.

The discussion that follows makes the assumption that the right hand side of every production is either a string of non-terminals or a single terminal. Each parsing operation generates a new element of structure, and so is associated with a position structure grammar production. The first type of parsing operation involves productions which generate a single terminal (e.g., $\mathrm{P} \Rightarrow \mathrm{p}$ ). Because we are assuming that an input segment may only be parsed into at most one position, and that a position may have at most one input segment parsed into it, this parsing operation may only fill a cell which covers exactly one input segment. in our example, cell $[\mathrm{P}, 1,1]$ could be filled by an operation parsing $i_{1}$ into a $\mathrm{p}$ position, giving the partial description $\mathrm{P}\left(\mathrm{p}\right.$ filled with $\left.i_{1}\right)$.

The other kinds of parsing operations are matched to position grammar productions in which a parent non-terminal generates child non-terminals. One of these kinds of operations fills the cell for a category by combining cell entries for two factor categories, in order, so that the substrings covered by each of them combine (concatenatively, with no overlap) to form the input substring covered by the cell being filled. For rule $\mathrm{Y} \Rightarrow \mathrm{MFM}$, there will be an operation of this type combining entries in $[\mathrm{M}, \mathrm{a}, \mathrm{b}]$ and $[\mathrm{F}, \mathrm{b}+1, \mathrm{c}]$, creating the concatenated structure $^{2}[\mathrm{M}, \mathrm{a}, \mathrm{b}]+[\mathrm{F}, \mathrm{b}+1, \mathrm{c}]$, to fill $[\mathrm{MF}, \mathrm{a}, \mathrm{c}]$. The final type of parsing operation fills a cell for a category which is a single non-terminal on the left hand side of a production, by combining two entries which jointly form the entire right hand side of the production. This operation would combining entries in $[\mathrm{MF}, \mathrm{a}, \mathrm{c}]$ and $[\mathrm{M}, \mathrm{c}+1, \mathrm{~d}]$, creating the structure $\mathrm{Y}([\mathrm{MF}, \mathrm{a}, \mathrm{c}],[\mathrm{M}, \mathrm{c}+1, \mathrm{~d}])$, to fill $[\mathrm{Y}, \mathrm{a}, \mathrm{d}]$. Each of these operations involves filling a cell for a target category by using the entries in the cells for two factor categories.

The resulting Harmony of the partial description created by a parsing operation will be the combina-

\footnotetext{
${ }^{2}$ This partial description is not a single tree, but an ordered pair of trees. In general, such concatenated structures will be ordered lists of trees.
}

tion of the marks assessed each of the partial descriptions for the factor categories, plus any additional marks incurred as a result of the structure added by the production itself. This is true because the constraints must be local: any new constraint violations are determinable on the basis of the cell category of the factor partial descriptions, and not any other internal details of those partial descriptions.

All possible ways in which the factor categories, taken in order, may combine to cover the substring, must be considered. Because the factor categories must be contiguous and in order, this amounts to considering each of the ways in which the substring can be split into two pieces. This is reflected in the parsing operation descriptions given in Section 6.2.

Underparsing operations are not matched with position grammar productions. A DP Table cell which covers only one input segment may be filled by an underparsing operation which marks the input segment as underparsed. In general, any partial description covering any substring of the input may be extended to cover an adjacent input segment by adding that additional segment marked as underparsed. Thus, a cell covering a given substring of length greater than one may be filled in two mirrorimage ways via underparsing: by taking a partial description which covers all but the leftmost input segment and adding that segment as underparsed, and by taking a partial description which covers all but the rightmost input segment and adding that segment as underparsed.

Overparsing operations are discussed in Section 5.

\section{The Overparsing Operations}

Overparsing operations consume no input; they only add new unfilled structure. Thus, a block of cells (the set of cells each covering the same input substring) is interdependent with respect to overparsing operations, meaning that an overparsing operation trying to fill one cell in the block is adding structure to a partial description from a different cell in the same block. The first consequence of this is that the overparsing operations must be considered after the underparsing and parsing operations for that block. Otherwise, the cells would be empty, and the overparsing operations would have nothing to add on to.

The second consequence is that overparsing operations may need to be considered more than once, because the result of one overparsing operation (if it fills a cell) could be the source for another overparsing operation. Thus, more than one pass through the overparsing operations for a block may be necessary. In the description of the algorithm given in Section 6.3 , each Repeat-Until loop considers the overparsing operations for a block of cells. The number of loop iterations is the number of passes through the overparsing operations for the block. The loop iterations stop when none of the overparsing operations 
is able to fill a cell (each proposed partial description is less harmonic than the partial description already in the cell).

In principle, an unbounded number of overparsing operations could apply, and in fact descriptions with arbitrary numbers of unfilled positions are contained in the output space of Gen (as formally defined). The algorithm does not have to explicitly consider arbitrary amounts of overparsing, however. A necessary property of the faithfulness constraints, given constraint locality, is that a partial description cannot have overparsed structures repeatedly added to it until the resulting partial description falls into the same cell category as the original prior to overparsing, and be more Harmonic. Such a sequence of overparsing operations can be considered a overparsing cycle. Thus, the faithfulness constraints must ban overparsing cycles. This is not solely a computational requirement, but is necessary for the grammar to be well-defined: overparsing cycles must be harmonically suboptimal, otherwise arbitrary amounts of overparsing will be permitted in optimal descriptions. In particular, the constraints should prevent overparsing from adding an entire overparsed nonterminal more than once to the same partial description while passing through the overparsing operations. In Example 2, the constraints FILL ${ }^{m}$ and FILL $^{p}$ effectively ban overparsing cycles: no matter where these constraints are ranked, a description containing an overparsing cycle will be less harmonic (due to additional FILL violations) than the same description with the cycle removed.

Given that the universal constraints meet this criterion, the overparsing operations may be repeatedly considered for a given level until none of them increase the Harmony of the entries in any of the cells. Because each overparsing operation maps a partial description in one cell category to one for another cell category, a partial description cannot undergo more consecutive overparsing operations than there are cell categories without repeating at least one cell category, thereby creating a cycle. Thus, the number of cell categories places a constant bound on the number of passes made through the overparsing operations for a block.

A single non-terminal may dominate an entire subtree in which none of the syllable positions at the leaves of the tree are filled. Thus, the optimal "unfilled structure" for each non-terminal, and in fact each cell category, must be determined, for use by the overparsing operations. The optimal overparsing structure for category $\mathrm{X}$ is denoted with $[\mathrm{X}, 0]$, and such an entity is referred to as a base overparsing structure. A set of such structures must be computed, one for each category, before filling input-dependent DP table cells. Because these values are not dependent upon the input, base overparsing structures may be computed and stored in advance. Computing them is just like computing other cell entries, except that only overparsing operations are considered. First, consider (once) the overparsing operations for each non-terminal $\mathrm{X}$ which has a production rule permitting it to dominate a terminal $\mathrm{x}$ : each tries to set $[\mathrm{X}, 0]$ to contain the corresponding partial description with the terminal $x$ left unfilled. Next consider the other overparsing operations for each cell, choosing the most Harmonic of those operations' partial descriptions and the prior value of $[\mathrm{X}, 0]$.

\section{The Dynamic Programming Algorithm}

\subsection{Notation}

$\operatorname{maxH}\{\}$ returns the argument with maximum Harmony

$\left\langle i_{a}\right\rangle$ denotes input segment $i_{a}$ underparsed

$\mathrm{X}^{t}$ is a non-terminal

$\mathrm{x}^{t}$ is a terminal

+ denotes concatenation

\subsection{The Operations}

Underparsing Operations for $\left[\mathrm{X}^{t}, \mathrm{a}, \mathrm{a}\right]$ : create $\left\langle i_{a}\right\rangle+\left[\mathrm{X}^{t}, 0\right]$

Underparsing Operations for $\left[\mathrm{X}^{t}, \mathrm{a}, \mathrm{c}\right]$ : create $\left\langle i_{a}\right\rangle+\left[\mathrm{X}^{t}, \mathrm{a}+1, \mathrm{c}\right]$

create $\left[\mathrm{X}^{t}, \mathrm{a}, \mathrm{c}-1\right]+\left\langle i_{a}\right\rangle$

Parsing operations for $\left[\mathrm{X}^{t}, \mathrm{a}, \mathrm{a}\right]$ : for each production $\mathrm{X}^{t} \Rightarrow \mathrm{x}^{k}$ create $\mathrm{X}^{t}\left(\mathrm{x}^{k}\right.$ filled with $\left.\mathrm{i}_{a}\right)$

Parsing operations for $\left[\mathrm{X}^{t}, \mathrm{a}, \mathrm{c}\right]$, where $c>a$ and all $X$ are cell categories:

for each production $\mathrm{X}^{t} \Rightarrow \mathrm{X}^{k} \mathrm{X}^{m}$

for $b=a+1$ to $c-1$ create $\mathrm{X}^{t}\left(\left[\mathrm{X}^{k}, \mathrm{a}, \mathrm{b}\right],\left[\mathrm{X}^{m}, \mathrm{~b}+1, \mathrm{c}\right]\right)$

for each production $\mathrm{X}^{u} \Rightarrow \mathrm{X}^{k} \mathrm{X}^{m} \mathrm{X}^{n} \ldots$ where $\mathrm{X}^{t}=\mathrm{X}^{k} \mathrm{X}^{m}$ :

for $b=a+1$ to $c-1$ create $\left[\mathrm{X}^{k}, \mathrm{a}, \mathrm{b}\right]+\left[\mathrm{X}^{m}, \mathrm{~b}+1, \mathrm{c}\right]$

Overparsing operations for $\left[\mathrm{X}^{t}, 0\right]$ :

for each production $\mathrm{X}^{t} \Rightarrow \mathrm{x}^{k}$ create $\mathrm{X}^{t}\left(\mathrm{x}^{k}\right.$ unfilled)

for each production $\mathrm{X}^{t} \Rightarrow \mathrm{X}^{k} \mathrm{X}^{m}$ create $\mathrm{X}^{t}\left(\left[\mathrm{X}^{k}, 0\right],\left[\mathrm{X}^{m}, 0\right]\right)$

for each production $\mathrm{X}^{u} \Rightarrow \mathrm{X}^{k} \mathrm{X}^{m} \mathrm{X}^{n} \ldots$ where $\mathrm{X}^{t}=\mathrm{X}^{k} \mathrm{X}^{m}$ : create $\left[\mathrm{X}^{k}, 0\right]+\left[\mathrm{X}^{m}, 0\right]$

Overparsing operations for $\left[\mathrm{X}^{t}, \mathrm{a}, \mathrm{a}\right]$ : same as for $\left[\mathrm{X}^{t}, \mathrm{a}, \mathrm{c}\right]$

Overparsing operations for $\left[\mathrm{X}^{t}, \mathrm{a}, \mathrm{c}\right]$ : for each production $\mathrm{X}^{t} \Rightarrow \mathrm{X}^{k}$ create $\mathrm{X}^{t}\left(\left[\mathrm{X}^{k}, \mathrm{a}, \mathrm{c}\right]\right)$ 
for each production $\mathrm{X}^{t} \Rightarrow \mathrm{X}^{k} \mathrm{X}^{m}$

create $\mathrm{X}^{t}\left(\left[\mathrm{X}^{k}, 0\right],\left[\mathrm{X}^{m}, \mathrm{a}, \mathrm{c}\right]\right)$

create $\mathrm{X}^{t}\left(\left[\mathrm{X}^{k}, \mathrm{a}, \mathrm{c}\right],\left[\mathrm{X}^{m}, 0\right]\right)$

for each production $\mathrm{X}^{u} \Rightarrow \mathrm{X}^{k} \mathrm{X}^{m} \mathrm{X}^{n} \ldots$

where $\mathrm{X}^{t}=\mathrm{X}^{k} \mathrm{X}^{m}$ :

create $\left[\mathrm{X}^{k}, \mathrm{a}, \mathrm{c}\right]+\left[\mathrm{X}^{m}, 0\right]$

create $\left[\mathrm{X}^{k}, 0\right]+\left[\mathrm{X}^{m}, \mathrm{a}, \mathrm{c}\right]$

\subsection{The Main Algorithm}

${ }^{*}$ create the base overparsing structures */

Repeat

For each $\mathrm{X}^{t}$, Set $\left[\mathrm{X}^{t}, 0\right]$ to

$\operatorname{maxH}\left\{\left[\mathrm{X}^{t}, 0\right]\right.$, overparsing ops for $\left.\left[\mathrm{X}^{t}, 0\right]\right\}$

Until no $\left[\mathrm{X}^{t}, 0\right]$ has changed during a pass

$/$ f fill the cells covering only a single segment */

For $\mathrm{a}=1$ to $\mathrm{J}$

For each $\mathrm{X}^{t}$, Set $\left[\mathrm{X}^{t}, \mathrm{a}, \mathrm{a}\right]$ to

$\operatorname{maxH}\left\{\right.$ underparsing ops for $\left.\left[\mathrm{X}^{t}, \mathrm{a}, \mathrm{a}\right]\right\}$

For each $\mathrm{X}^{t}$, Set $\left[\mathrm{X}^{t}, \mathrm{a}, \mathrm{a}\right]$ to

$\operatorname{maxH}\left\{\left[\mathrm{X}^{t}, \mathrm{a}, \mathrm{a}\right]\right.$, parsing ops for $\left.\left[\mathrm{X}^{t}, \mathrm{a}, \mathrm{a}\right]\right\}$

Repeat

For each $\mathrm{X}^{t}$, Set $\left[\mathrm{X}^{t}, \mathrm{a}, \mathrm{a}\right]$ to

$\operatorname{maxH}\left\{\left[\mathrm{X}^{t}, \mathrm{a}, \mathrm{a}\right]\right.$, overparsing ops for $\left.\left[\mathrm{X}^{t}, \mathrm{a}, \mathrm{a}\right]\right\}$

Until no $\left[\mathrm{X}^{t}, \mathrm{a}, \mathrm{a}\right]$ has changed during a pass

$/^{*}$ fill the rest of the cells */

For $\mathrm{d}=1$ to $(\mathrm{J}-1)$

For $a=1$ to $(J-d)$

For each $\mathrm{X}^{t}$, Set $\left[\mathrm{X}^{t}, \mathrm{a}, \mathrm{a}+\mathrm{d}\right]$ to

$\operatorname{maxH}\left\{\right.$ underparsing ops for $\left.\left[\mathrm{X}^{t}, \mathrm{a}, \mathrm{a}+\mathrm{d}\right]\right\}$

For each $\mathrm{X}^{t}$, Set $\left[\mathrm{X}^{t}, \mathrm{a}, \mathrm{a}+\mathrm{d}\right]$

$\operatorname{maxH}\left\{\left[\mathrm{X}^{t}, \mathrm{a}, \mathrm{a}+\mathrm{d}\right]\right.$, parsing ops for $\left.\left[\mathrm{X}^{t}, \mathrm{a}, \mathrm{a}+\mathrm{d}\right]\right\}$

Repeat

For each $\mathrm{X}^{t}$,

Set $\left[\mathrm{X}^{t}, \mathrm{a}, \mathrm{a}+\mathrm{d}\right]$ to

$\operatorname{maxH}\left\{\left[\mathrm{X}^{t}, \mathrm{a}, \mathrm{a}+\mathrm{d}\right]\right.$,

overparsing ops for $\left.\left[\mathrm{X}^{t}, \mathrm{a}, \mathrm{a}+\mathrm{d}\right]\right\}$

Until no $\left[\mathrm{X}^{t}, \mathrm{a}, \mathrm{a}+\mathrm{d}\right]$ has changed during a pass

Return $[\mathrm{S}, 1, \mathrm{~J}]$ as the optimal description

\subsection{Complexity}

Each block of cells for an input subsequence is processed in time linear in the length of the subsequence. This is a consequence of the fact that in general parsing operations filling such a cell must consider all ways of dividing the input subsequence into two pieces. The number of overparsing passes through the block is bounded from above by the number of cell categories, due to the fact that overparsing cycles are suboptimal. Thus, the number of passes is bounded by a constant, for any fixed position structure grammar. The number of such blocks is the number of distinct, contiguous input subsequences (equivalently, the number of cells in a layer), which is on the order of the square of the length of the input. If $\mathrm{N}$ is the length of the input, the algorithm has computational complexity $\mathrm{O}\left(\mathrm{N}^{3}\right)$.

\section{Discussion}

\subsection{Locality}

That locality helps processing should be no great surprise to computationalists; the computational significance of locality is widely appreciated. Further, locality is often considered a desirable property of principles in linguistics, independent of computational concerns. Nevertheless, locality is a sufficient but not necessary restriction for the applicability of this algorithm. The locality restriction is really a special case of a more general sufficient condition. The general condition is a kind of "Markov" property. This property requires that, for any substring of the input for which partial descriptions are constructed, the set of possible partial descriptions for that substring may be partitioned into a finite set of classes, such that the consequences in terms of constraint violations for the addition of structure to a partial description may be determined entirely by the identity of the class to which that partial description belongs. The special case of strict locality is easy to understand with respect to context-free structures, because it states that the only information needed about a subtree to relate it to the rest of the tree is the identity of the root non-terminal, so that the (necessarily finite) set of non-terminals provides the relevant set of classes.

\subsection{Underparsing and Derivational Redundancy}

The treatment of the underparsing operations given above creates the opportunity for the same partial description to be arrived at through several different paths. For example, suppose the input is $i_{a} \ldots i_{b} i_{c} i_{d} \ldots i_{e}$, and there is a constituent in [X, a, b] and a constituent [Y,d,e]. Further suppose the input segment $i_{c}$ is to be marked underparsed, so that the final description $[\mathrm{S}, \mathrm{a}, \mathrm{e}]$ contains $[\mathrm{X}, \mathrm{a}, \mathrm{b}]\left\langle i_{c}\right\rangle[\mathrm{Y}, \mathrm{d}, \mathrm{e}]$. That description could be arrived at either by combining $[\mathrm{X}, \mathrm{a}, \mathrm{b}]$ and $\left\langle i_{c}\right\rangle$ to fill $[\mathrm{X}, \mathrm{a}, \mathrm{c}]$, and then combine $[X, a, c]$ and $[Y, d, e]$, or it could be arrived at by combining $\left\langle i_{c}\right\rangle$ and $[\mathrm{Y}, \mathrm{d}, \mathrm{e}]$ to fill $[\mathrm{Y}, \mathrm{c}, \mathrm{e}]$, and then combine $[\mathrm{X}, \mathrm{a}, \mathrm{b}]$ and $[\mathrm{Y}, \mathrm{c}, \mathrm{e}]$. The potential confusion stems from the fact that an underparsed segment is part of the description, but is not a proper constituent of the tree.

This problem can be avoided in several ways. An obvious one is to only permit underparsings to be added to partial descriptions on the right side. One exception would then have to be made to permit input segments prior to any parsed input segments to be underparsed (i.e., if the first input segment is underparsed, it has to be attached to the left side of some constituent because it is to the left of everything in the description). 


\section{Conclusions}

The results presented here demonstrate that the basic cubic time complexity results for processing context-free structures are preserved when Optimality Theory grammars are used. If Gen can be specified as matching input segments to structures generated by a context-free position structure grammar, and the constraints are local with respect to those structures, then the algorithm presented here may be applied directly to compute optimal descriptions.

\section{Acknowledgments}

I would like to thank Paul Smolensky for his valuable contributions and support. I would also like to thank David Haussler, Clayton Lewis, Mark Liberman, Jim Martin, and Alan Prince for useful discussions, and three anonymous reviewers for helpful comments. This work was supported in part by an NSF Graduate Fellowship to the author, and NSF grant IRI-9213894 to Paul Smolensky and Geraldine Legendre.

\section{References}

S. O. Anderson and R. C. Backhouse. 1981. Locally least-cost error recovery in Earley's algorithm. ACM Transactions on Programming Languages and Systems 3: 318-347.

Eugene Charniak. 1993. Statistical language learning. Cambridge, MA: MIT Press.

Martin Kay. 1980. Algorithmic schemata and data structures in syntactic processing. CSL-80-12, October 1980 .

K. Lari and S. J. Young. 1990. The estimation of stochastic context-free grammars using the insideoutside algorithm. Computer Speech and Language 4: 35-36.

Harry R. Lewis and Christos H. Papadimitriou. 1981. Elements of the theory of computation. Englewood Cliffs, New Jersey: Prentice-Hall, Inc.

Alan Prince and Paul Smolensky. 1993. Optimality Theory: Constraint interaction in generative grammar. Technical Report CU-CS-696-93, Department of Computer Science, University of Colorado at Boulder, and Technical Report TR-2, Rutgers Center for Cognitive Science, Rutgers University, New Brunswick, NJ. March. To appear in the Linguistic Inquiry Monograph Series, Cambridge, MA: MIT Press.

David Sankoff and Joseph Kruskal. 1983. Time warps, string edits, and macromolecules: The theory and practice of sequence comparison. Reading, MA: Addison-Wesley.
Bruce Tesar. 1994. Parsing in Optimality Theory: A dynamic programming approach. Technical Report CU-CS-714-94, April 1994. Department of Computer Science, University of Colorado, Boulder.

Bruce Tesar. 1995a. Computing optimal forms in Optimality Theory: Basic syllabification. Technical Report CU-CS-763-95, February 1995. Department of Computer Science, University of Colorado, Boulder.

Bruce Tesar. 1995b. Computational Optimality Theory. Unpublished Ph.D. Dissertation. Department of Computer Science, University of Colorado, Boulder. June 1995.

A.J. Viterbi. 1967. Error bounds for convolution codes and an asymptotically optimal decoding algorithm. IEEE Trans. on Information Theory 13:260-269. 